\title{
Nicole Laurin
}

sociologue, département de sociologie, Université de Montréal

(2005)

\section{"Genèse de la sociologie marxiste au Québec"}

Un document produit en version numérique par Jean-Marie Tremblay, bénévole, professeur de sociologie au Cégep de Chicoutimi

Courriel: jean-marie tremblay@uqac.ca

Site web pédagogique : http://www.uqac.ca/jmt-sociologue/

Dans le cadre de la collection: "Les classiques des sciences sociales" Site web: http://classiques.uqac.ca/

Une collection développée en collaboration avec la Bibliothèque Paul-Émile-Boulet de l'Université du Québec à Chicoutimi Site web: http://bibliotheque.uqac.ca/ 
Cette édition électronique a été réalisée par Jean-Marie Tremblay, bénévole, professeur de sociologie au Cégep de Chicoutimi à partir de :

Nicole Laurin-Frenette

“Genèse de la sociologie marxiste au Québec".

Un article publié dans la revue Sociologie et sociétés, vol. 37, no 2, automne 2005, pp. 183-207. Montréal: Les Presses de l'Université de Montréal.

M Laurin, sociologue, professeure au département de sociologie de l'Université de Montréal, nous a accordé le 14 janvier 2003 son autorisation de diffuser électroniquement toutes ses œuvres dans Les Classiques des sciences sociales.

Courriel : laurinn@SOCIO.UMontreal.CA

Polices de caractères utilisée :

Pour le texte: Times New Roman, 14 points.

Pour les citations : Times New Roman, 12 points.

Pour les notes de bas de page : Times New Roman, 12 points.

Édition électronique réalisée avec le traitement de textes Microsoft Word 2004 pour Macintosh.

Mise en page sur papier format : LETTRE (US letter), 8.5” x 11")

Édition numérique réalisée le 11 décembre 2006 à Chicoutimi, Ville de Saguenay, province de Québec, Canada.

\section{Fait avec}


Nicole Laurin

"Genèse de la sociologie marxiste au Québec"
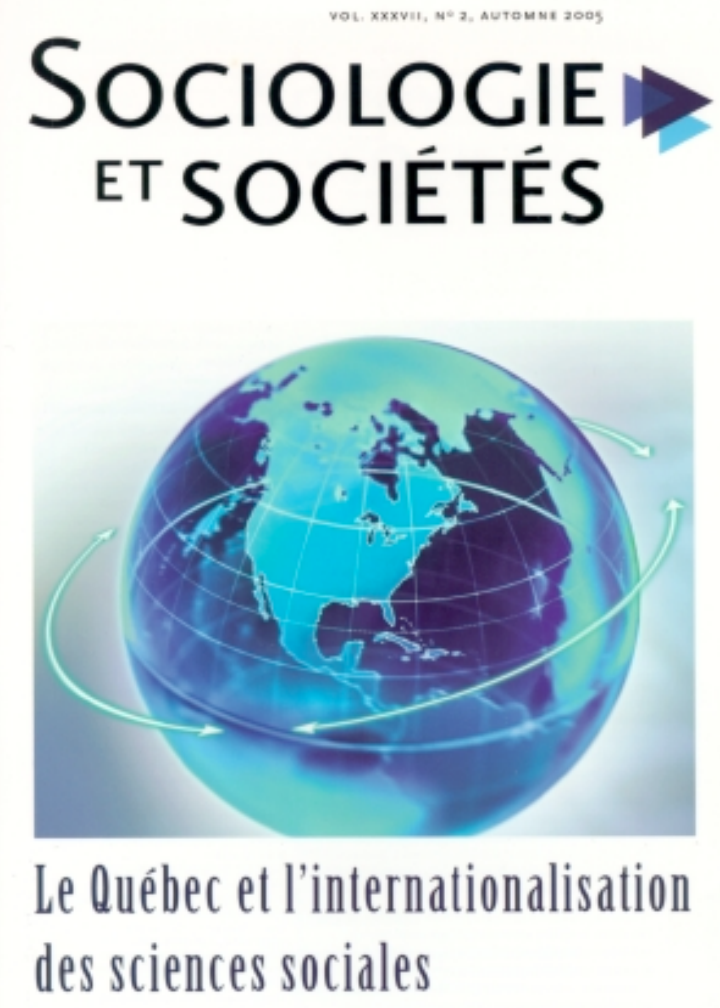

Les Presses de I'Université de Montréal

Un article publié dans la revue Sociologie et sociétés, vol. 37, no 2, automne 2005, pp. 183-207. Montréal: Les Presses de l'Université de Montréal. 


\section{Table des matières}

$\underline{\text { Introduction }}$

PARTI PRIS ET LE MARXISME, 1963-1968

LA REVUE SOCIALISME, 1964-1974

Résumé

BIBLIOGRAPHIE 
Nicole Laurin

\section{“Genèse de la sociologie marxiste au Québec". 1}

Un article publié dans la revue Sociologie et sociétés, vol. 37, no 2, automne 2005, pp. 183-207. Montréal: Les Presses de l'Université de Montréal.

\section{Introduction}

$\underline{\text { Retour à la table des matières }}$

1 NOTE DE L'AUTEURE : Les responsables de ce numéro m'ont demandé d'étudier la sociologie marxiste au Québec. À cette fin, j'ai relu les nombreux essais et les recueils de textes qui se situent dans ce courant. Au hasard de mes recherches, je suis tombée sur quelques numéros de Parti pris et de Socialisme dont la lecture m'a fascinée. Dès le début des années 1960, de très jeunes gens élaborent dans ces revues une pensée critique, originale, sur le Québec et sa place dans le monde. À la recherche d'un cadre et d'un langage, ils découvrent le marxisme et l'adaptent à leur usage. Parmi ces jeunes, plusieurs sont sociologues et deviendront professeurs. Ils posent un regard critique sur les travaux des intellectuels qui ont été des précurseurs du marxisme au Québec et dont certains ont fondé Socialisme. D'abord tiers-mondistes, les jeunes sociologues s'approprient les œuvres classiques du marxisme ; ils se penchent aussi sur les publications récentes des intellectuels français marxistes ou marxisants. Enthousiasmés par la nouvelle interprétation structuraliste de Marx, ils deviennent althussériens. Ainsi, entre 1963 et 1974, un marxisme « fait au Québec » sinon « québécois » va prendre forme. Axé sur l'étude de la question nationale, les classes sociales et la révolution. J'ai voulu retracer cette histoire en détail, sans méthode analytique et sans théorie. Mon texte est un récit. Il campe des personnages. L'intrigue se noue autour de questions théoriques et politiques. L'action se déroule sur la scène universitaire, elle s'inscrit également dans le débat public et les luttes sociales. Ce genre de travail n'a sans doute pas sa place dans une revue universitaire de sociologie. La direction de Sociologie et sociétés a bien voulu l'accepter. Je la remercie. je suis aussi reconnaissante à Alfred Dubuc, Luc Racine et Céline Saint-Pierre pour leur précieuse collaboration à mes recherches. 
Avant la décennie 1960, dans le Québec francophone, la pensée de Marx et des auteurs marxistes n'est pas absente de l'enseignement universitaire mais elle sert essentiellement de repoussoir à la doctrine sociale de l'Église. Le marxisme ne fait pas partie des théories agréées par l'autorité catholique ; il est condamné parce qu'il est athée et fondé sur les postulats philosophiques du matérialisme. Il prône en plus la lutte des classes et la révolution prolétarienne dans le but d'instaurer le communisme. On peut faire l'hypothèse que jusqu'aux années de la Révolution tranquille, les principaux lecteurs de l'oeuvre de Marx et ses véritables connaisseurs furent des clercs : de savants pères jésuites ou dominicains, titulaires de l'enseignement supérieur ou responsables de l'encadrement pastoral des organisations syndicales, des associations étudiantes et autres. À l'époque, l'Église détient l'hégémonie sur les universités de langue française. Elles sont régies par des chartes pontificales et les recteurs sont des évêques. Un grand nombre de clercs y dispensent des enseignements dans différents domaines, $\mathrm{y}$ compris en sciences sociales. Stanley B. Ryerson, historien marxiste éminent et membre du Parti communiste canadien, raconte que lors du congrès des Sociétés savantes du Canada, tenu à l'Université Laval, en 1963, les autorités de cette institution firent retirer d'une exposition de livres un étalage d'ouvrages marxistes dont le sien. Selon Ryerson, des thèmes marxistes avaient été discutés au cours de ce congrès (Ryerson, 1965). Néanmoins, au cours des années de la Révolution tranquille, la liberté de pensée et d'expression gagne sans cesse du terrain. L'emprise de l'Église sur les esprits diminue et l'Église elle-même se rénove. Ainsi, la société s'ouvre à de nouveaux courants d'idées; des groupes et des mouvements politiques ou culturels s'organisent, des revues voient le jour. Deux générations d'intellectuels marxistes de langue française entrent alors en scène, presque simultanément. La première génération est celle des professeurs, au demeurant peu nombreux, qui ont étudié en Europe et sont sensibilisés aux idées de la gauche. Ils se trouvent principalement à l'Université de Montréal, où ils contribuent à initier au marxisme une première cohorte d'étudiants en sciences sociales, notamment en sociologie. Parmi ces derniers, plusieurs entrent très tôt dans les rangs de l'enseignement universitaire, alors en pleine expansion. En effet, l'Université du Québec à Montréal, fondée en 1969, recrute une grande partie de la génération montante. Ces jeunes professeurs viennent tout juste d'achever ou ils achèveront quelques années plus tard leur formation à l'étranger, en 
France principalement. Dès lors, deux départements de sociologie assurent au premier chef le développement et l'essor du marxisme au Québec : le département de l'Université de Montréal, au cours des années 1960, relayé par celui de l'UQÀM, au cours des années 1970. Cette sociologie marxiste intègre des travaux d'historiens, de politologues, d'économistes et autres spécialistes des sciences sociales. Fidèles à la tradition, ses adeptes n'hésitent pas à s'engager dans l'action politique. Pour retracer sa genèse au Québec, deux revues serviront de fil conducteur : Parti pris et Socialisme. En 1975, Marcel Rioux évoque leur naissance en ces termes : «Un soir de 1963, Vadeboncoeur, Dofny et moi-même rencontrâmes des jeunes qui, comme nous, voulaient fonder une revue ; ils s'appelaient Chamberland, Maheu, Major et Piotte ; nous devions lancer Socialisme 64 quelques mois après qu'ils eurent fondé Parti pris. À cette réunion, nous avons constaté que tout en combattant pour les mêmes objectifs - un Québec libre et socialiste -, nous visions des publics différents, avec des ressources et des moyens eux aussi différents »(Rioux, 1975, pp. 5-6). On retracera d'abord le parcours de la revue Parti pris, de 1963 à 1968. Elle peut être considérée comme le berceau de la jeune génération de marxistes. On suivra ensuite l'évolution de la revue Socialisme, au cours de ses dix années de publication. Fondée en 1964 par la première génération de marxistes, elle tombe quatre ans plus tard entre les mains de la jeune génération, qui la dirige jusqu'en 1974.

\section{PARTI PRIS ET LE MARXISME, 1963-1968}

\section{$\underline{\text { Retour à la table des matières }}$}

À ses débuts, en 1963, la revue Parti pris réunit des jeunes qui se déclarent révolutionnaires. Étudiants, employés, artistes, ils ont vingt ans ou à peine plus. Plus tard, certains feront carrière à l'université, d'autres dans les lettres et les arts. Chaque numéro de Parti pris comporte deux volets : le premier consacré à l'analyse et à la discussion de questions sociales et politiques, le second réservé à des chroniques de l'art et de la culture : roman, poésie, musique, cinéma, etc. Entre 1963 
et 1968 , le comité de rédaction de la revue se compose, entre autres, de Paul Chamberland, Pierre Maheu, Jean-Marc Piotte, Gaétan Tremblay, Luc Racine, Gabriel Gagnon, Patrick Straram, qui en forment le noyau stable. Plusieurs collaborateurs réguliers ou ponctuels se joignent à eux, à différentes étapes. D'emblée, les fondateurs de la revue s'affirment « socialistes, laïcistes et indépendantistes »(Parti pris, 1963, p. 3). À leurs yeux, le Québec est une société colonisée. Elle ressemble sous divers aspects aux pays du tiers-monde qui luttent à cette époque pour leur indépendance. Pour parler de la révolution au Québec, Parti pris emprunte le vocabulaire de la décolonisation, puisé dans les textes du mouvement tiers-mondiste. Dans l'éditorial du numéro de février 1964, " Nous avons choisi la révolution », l'équipe de la revue souhaite la formation d'un parti révolutionnaire, qui s'appuie sur les travailleurs (Parti pris, 1964a). Elle prône « une praxis révolutionnaire cohérente et généralisée », affirme son refus de l'ordre qui serait « une première brèche dans ce mur qui nous sépare des exploités »(Parti pris, 1964a, p. 5). Certains membres de la revue s'interrogent sur les contradictions de la Révolution tranquille, alors en cours, dans des termes qui évoquent déjà la problématique marxiste. Le nationalisme qui alimente le discours de la Révolution tranquille et justifie ses réalisations ne serait-il pas l'idéologie d'une nouvelle bourgeoisie québécoise ? Or, l'intérêt de cette classe n'est pas nécessairement celui de toute la nation, à s'oppose même le plus souvent à l'intérêt des classes populaires. Dès lors, le deuxième moment de cette révolution serait-il la prise du pouvoir par les travailleurs, les classes dites exploitées et aliénées ? Au cours des deux décennies à venir, les marxistes québécois francophones, de toutes tendances, vont débattre ces deux questions énoncées par Parti pris : la question théorique des relations entre les classes sociales et la nation, et la question politique du rapport entre la libération nationale et la libération sociale.

La revue se penche sur les conditions de vie et de travail des classes populaires. Plusieurs textes traitent du milieu ouvrier ou du milieu rural, des orientations du syndicalisme ouvrier ou agricole, des associations étudiantes. À cette époque, le Bureau d'aménagement de l'Est du Québec, créé par le gouvernement québécois, suscite l'intérêt de la gauche, notamment de Parti pris, parce qu'il mise sur la participation active de la population à la planification et la réalisation d'un projet de développement régional. Certains observateurs, à Parti pris et dans 
d'autres milieux, croient d'ailleurs que la révolution pourrait venir des régions rurales plutôt que de Montréal. D'autre part, Parti pris a pour toile de fond les groupes et les partis politiques de formation récente : le Rassemblement pour l'indépendance nationale, le Parti socialiste du Québec et le Front de libération du Québec - organisation clandestine qui privilégie l'action terroriste. Au fil des années, la revue observe et commente leur évolution idéologique et leur position politique sur différentes questions. Elle critique sévèrement ces formations, les jugeant en général peu aptes à favoriser la révolution. Sans être indulgente à l'endroit du FLQ, elle n'est pas excessivement sévère. Elle publie occasionnellement les lettres des felquistes Raymond Villeneuve, Pierre Vallières et Charles Gagnon. Ce dernier collabore régulièrement à la revue, au cours de ses années d'emprisonnement. En 1964, Parti pris fonde le Mouvement de libération populaire en espérant qu'il devienne un parti révolutionnaire, l'instrument de la prise du pouvoir par les travailleurs. En 1966, le MLP et la revue adhèrent en bloc au PSQ, formant ainsi la gauche de ce parti, ancré dans le mouvement syndical, et qui prône un socialisme modéré. Mais le PSQ n'en a pas pour longtemps à vivre; il se disloquera en 1968. Parmi les pouvoirs en place, les cibles privilégiées de Parti pris sont non seulement l'État, fédéral ou provincial, mais également l'Église. En effet, l'éradication du cléricalisme et l'instauration de la laïcité forment la thématique de plusieurs articles et d'un numéro spécial, intitulé "Bilan du cléricalisme »(Parti pris, 1966). Au sein de l'équipe, Pierre Maheu s'intéresse tout particulièrement à la critique de l'Église et de son rôle dans la société québécoise. En décembre 1966, la revue organise aussi un colloque sur le thème "Le dieu canadien-français contre l'homme québécois », dans le cadre du « teach-in laïque » de l'Université de Montréal (Parti pris, 1966, p. 3 de couverture).

Parti pris ne s'oppose pas aux autres revues de la gauche mais elle s'en démarque et les critique à l'occasion. Ainsi, Piotte écrit que les cité-libristes sont « des intellectuels bourgeois », et il se moque de leur philosophie humaniste, qu'il qualifie de "pseudo-personnalisme » (Piotte, 1964a, p. 4). Gérard Pelletier et Pierre Trudeau ayant accusé Parti Pris de nationalisme, se font répondre par Camille Limoges que "l'homme citoyen ne peut être détaché de "l'homme concret", qui prend racine dans son milieu » (Limoges, 1964, p. 3). À ses débuts, la nouvelle revue Socialisme 64, fondée par des syndicalistes de la CSN 
et de la FTQ et par des professeurs de l'Université de Montréal, ne suscite aucun enthousiasme. Au contraire, Jean-Marc Piotte se dit déçu par le premier numéro. Il aurait espéré, écrit-il, trouver des idées neuves mais « hélas! le manque d'originalité composait avec une tenue générale - un style quoi - qui sentait le musée, le vieilli » (Piotte, 1964b, p. 37). Il affirme par ailleurs que « Parti pris a appris de Liberté » (Piotte, 1964a, p. 4). Un des artisans de cette revue, Michel Van Schendel, établit le contact avec Parti pris (Dostaler, 2001, p. 467). Il y publie d'abord un poème et, en mars 1964, un article étonnant: « La maladie infantile du Québec » (Van Schendel, 1964a). " Le mot Québec, écrit Van Schendel, est depuis 1837 le nom d'une maladie » (Van Schendel, 1964a, p. 25). Il multiplie par la suite les termes dépréciatifs pour décrire ce pays et ses habitants : " colonisé ", " isolé dans une réserve coloniale », " sous-développés », " dépossédés ", " conscience aliénée », « capitalisme bâtard », « fédéralisme boiteux », etc. (Van Schendel, 1964a, pp. 25-45). L'auteur est marxiste, il est devenu membre du Parti communiste, en France, à la fin des années 1940. Il vit au Québec depuis 1952. Détenteur d'une double formation en lettres et en sciences économiques, il enseigne la littérature à l'Université de Montréal et devient, en 1969, professeur au Département d'études littéraires de l'UQÀM (Chamberland et al., 2001). Il est aussi poète, auteur d'une oeuvre littéraire importante. Dès 1966, il se joint à la revue Socialisme et, plus tard, il recrute les jeunes marxistes, sur lesquels il exercera une grande influence.

En 1964, ceux-ci sont encore à Parti pris, militant pour ce qu'ils désignent comme un " socialisme décolonisateur ", dont la forme cependant demeure imprécise. Aussi se mettent-ils à la recherche d'une pensée apte à guider leurs analyses et orienter leur stratégie. Sous la plume de Jean-Marc Piotte, l'éditorial du numéro de mars 1964 annonce que «Parti pris se rattache à la tradition marxiste-léniniste car nous la jugeons la moins dépassée par l'évolution historique et la plus apte, actuellement, à résoudre les problèmes que soulève un système inhumain, le système de la "free enterprise" [...] Le marxisme est fondamentalement une méthode qui nous permet d'analyser et de totaliser la société dans son dynamisme et son historicité » (Piotte, 1964a, p. 2). D'autre part, dans le numéro de septembre 1964, Piotte souligne que certains collaborateurs de Parti pris ont été formés à l'école de Sartre et que la revue a aussi « adapté à la réalité québécoise... les 
conceptions de Memmi, Fanon, Berque, Marx et Lénine " (Piotte, 1964b, p. 37). Les trois premiers auteurs sont des théoriciens de la décolonisation, dont les travaux s'inspirent notamment de l'expérience vécue par les populations du Maghreb. Leurs problématiques sont axées sur les thèmes de la dépossession et de la domination. L'anthropologue français, Jacques Berque, s'intéresse d'ailleurs à Parti pris. Il envoie une lettre aux membres de la rédaction et quelques textes qui seront publiés au cours des années suivantes. Pour sa part, Paul Chamberland propose, dans un éditorial intitulé «La révolution, c'est le peuple ", de traduire en termes d'éthique, l'action politique de la gauche (Chamberland, 1964). Il insiste sur la nécessité pour les révolutionnaires «d'être en osmose avec le peuple » : il faut, écrit-il, " écouter, apprendre du peuple, puis... lui donner voix » (Chamberland, 1964, p. 5). Ces principes, inspirés de la tradition anarchiste, suscitent toutefois peu d'intérêt à Parti pris. Au cours des années 1965 et 1966, les membres de la revue se forment au marxisme. L'expression « matérialisme historique et dialectique " apparaît. D'un numéro à l'autre, leur vocabulaire s'enrichit de termes marxistes, la plupart du temps sans référence précise. Leur connaissance de la théorie progresse. Puisant leurs idées dans les textes récents d'intellectuels français marxistes ou marxisants, ils ne s'intéressent pas encore aux classiques. Par exemple, Jean-Marc Piotte compare les vues de Serge Mallet et d'André Gorz sur les rapports entre réforme et révolution (Piotte, 1965a). Michel Mill reprend la discussion de cette théorie de Gorz dans le numéro suivant (Mill, 1965). Pierre Maheu aborde le thème de la justice de classe en s'appuyant sur une conception marxiste du droit dont les notions proviennent de deux articles, l'un de Nicos Poulantzas et l'autre de Jean-Marie Vincent (Maheu, 1965). De son côté, Piotte a découvert le Traité d'économie marxiste du trotskiste Ernest Mandel dont la lecture, affirme-t-il, lui permet de comprendre Marx (Motte, 1965b). Il n'a jamais lu Le Capital, jugé trop difficile, mais il insiste néanmoins sur le fait que sa " vision du monde » prend sa source désormais dans le « marxisme-léninisme » (Motte, 1965b, p. 109). André Pollender s'appuie sur l'économiste marxiste français Henri Denis dans un article sur la planification en URSS (Pollender, 1965). Les théories économiques de Marx, telles qu'appliquées en URSS, sont exposées par Georges Kostakeff dans "Marxisme et créditisme " (Kostakeff, 1966). Par ailleurs, au cours d'une crise qui secoue l'Association des étudiants de l'Université de Montréal, Parti pris brandit le 
célèbre pamphlet de Lénine, La maladie infantile du communisme, le gauchisme, pour condamner l'attitude des étudiants (Trudel, 1965, p. 5). Dans la même veine, un article bien étayé de René Beaudin, intitulé «Critique de la stratégie anarchiste », puise ses arguments dans des textes de Lénine et de Lukacs (Beaudin, 1966).

En mars 1966, un débat important oppose Paul Chamberland à Jean-Marc Piotte sur le fondement de la morale révolutionnaire. Au point de départ, ils rejettent d'un commun accord la philosophie humaniste, représentée en l'occurrence par l'ouvrage récent de Pierre Vadeboncoeur, L'autorité du peuple. Selon Motte, il existe une "base scientifique de la morale du matérialisme historique » (Piotte, 1966a, p. 3). Le devoir de faire la révolution s'impose lorsque les conditions objectives y sont propices. Il appuie son argumentation sur l'interprétation que fait Gramsci de certains passages de l'œuvre de Marx. La pensée de Gramsci sera le sujet de la thèse de doctorat de Piotte, dirigée par Lucien Goldman. Chamberland s'inspire par contre d'une conception de la révolution qui met davantage l'accent sur la transformation de la vie quotidienne et de la conscience. Plus tard, Chamberland s'engagera à fond dans le mouvement de la contre-culture ; il produira aussi une oeuvre littéraire remarquable. Ce débat amorce un changement d'orientation de la revue qui devient visible à l'automne suivant. Désormais, il ne suffit plus de connaître le marxisme, il faut le mettre en pratique. Dans l'éditorial intitulé « Exigences théoriques d'un combat politique ", c'est Paul Chamberland, paradoxalement, qui déplore le manque de rigueur du marxisme de Parti pris, lequel en serait resté aux bonnes intentions (Chamberland, 1966a). Or, selon lui, seul le développement de la pratique théorique peut guider l'action. La première tâche de la revue est donc de définir le socialisme décolonisateur qui représente l'objectif de la révolution. Le thème de la pratique théorique est emprunté à l'école althussérienne. Les althussériens proposent une interprétation structuraliste de Marx. Ils appliquent au travail intellectuel aussi bien qu'au travail manuel les trois notions - matière première, moyen de production, produit - qui désignent chez Marx les éléments du procès de production. Chamberland se réfère d'ailleurs explicitement à l'ouvrage de Louis Althusser, Pour Marx. C'est la première mention dans la revue de ce nouveau courant du marxisme, dont plusieurs auteurs se rattachent au Parti communiste français. Leurs idées se propagent très rapidement dans la jeune génération de 
marxistes. Elles demeureront sa principale source d'inspiration, pendant plus d'une décennie. Toutefois, dans le même numéro, celui de septembre et octobre 1966, Gabriel Gagnon affirme sans ambages préférer les thèses du socialisme autogestionnaire à celles du communisme orthodoxe (Gagnon G., 1966). Gagnon est un jeune anthropologue qui s'est joint depuis peu à la revue. Formé à l'Université Laval, il a fait sa thèse de doctorat en France, avec Georges Balandier. Il enseigne au Département de sociologie de l'Université de Montréal. Très proche de Marcel Rioux, il privilégie comme lui les thèmes libertaires de la pensée de Marx. Sous la plume de Gagnon, ces thèmes referont surface dans la revue, à différents moments.

En 1967, Parti pris est une revue marxiste. De plus, elle présente la qualité et la rigueur intellectuelles d'une publication universitaire. Elle le doit à plusieurs jeunes sociologues, formés à l'Université de Montréal, qui sont devenus membres de la rédaction. Parmi ceux-ci, Gilles Bourque, Gilles Dostaler, Michel Pichette, Narciso Pizarro, venus se joindre à Gabriel Gagnon et Luc Racine. Racine est l'assistant de recherche de Marcel Rioux au Département de sociologie ; il est aussi poète et musicien. D'autres jeunes sociologues apportent une contribution ponctuelle à la revue : Jean-Louis Chabot, Charles Gagnon, Nicole Gagnon, Lise Rochon, Ronald Sabourin. Plusieurs ont reçu aussi une formation en anthropologie, en histoire, en économie, en philosophie et même en sciences. Ils publient dans Parti pris des analyses marxistes de la société québécoise d'une remarquable originalité, sans succomber à ce stade au dogmatisme et au jargon d'école. Ils entendent produire et diffuser une connaissance rigoureuse de la réalité sociale, capable d'éclairer l'engagement et l'action politiques -ce dont rêvent d'ailleurs nombre d'adeptes des sciences sociales depuis Auguste Comte. Ainsi, Luc Racine écrit : " l'avènement du socialisme, au Québec comme ailleurs, dépend entre autres choses de la rencontre de la pratique socialiste et du savoir scientifique » (Racine, 1967a, p. 94). Dans ce contexte, l'étude des mouvements et des formations politiques demeure l'un des thèmes importants de la revue. On insiste sur leur composition de classes, sur les alliances et les luttes de classes en leur sein. Une recherche exhaustive sur le syndicalisme québécois -ouvrier, agricole, étudiant - est réalisée collectivement, dans le cadre de cette problématique (Parti pris, 1967a). Dans la même livraison, Gabriel Gagnon se penche sur la théorie de l'autogestion et il examine les mo- 
dalités de la pratique autogestionnaire dans différents domaines (Gagnon G., 1967). À l'occasion d'un compte rendu de l'ouvrage de Fernand Ouellet, Histoire économique et sociale du Québec, 1760-1850, Gilles Bourque et Luc Racine proposent une nouvelle explication de l'insurrection de 1837-1838 (Bourque et Racine, 1967). Elle aurait opposé ce que les auteurs appellent des ensembles de classes - nation ou société - : un premier ensemble à fondement socio-économique progressif à un second ayant un fondement économique attardé, résultat de la domination d'une société par une autre. Gilles Bourque a fait d'ailleurs son mémoire de maîtrise sur ce sujet. Luc Racine recense aussi le plus récent ouvrage du politologue Gérard Bergeron, auquel il oppose sa propre conception théorique de l'État (Racine, 1967a). Les jeunes sociologues ne se contentent pas de critiquer, ils proposent des idées originales, qu'ils ont élaborées ensemble. Ils partagent leurs lectures et leurs recherches; ils cosignent leurs textes.

Le dernier numéro de 1967 est consacré au thème «Aliénation et dépossession » (Parti pris, 1967b). Il marque un point de rupture dans la démarche de Parti pris. Dans la présentation du numéro, Luc Racine déclare que le langage de la colonisation et de la décolonisation n'est plus pertinent (Racine, 1967b). Il explique que les artisans de la revue sont passés désormais de l'humanisme à l'analyse scientifique, suivant une démarche analogue à celle qui a conduit Marx des Manuscrits économico-philosophiques au Capital. " Nous apprenons maintenant, écrit Racine, à démonter péniblement les mécanismes socio-économiques d'une domination totale, à expliquer notre mal après l'avoir nommé » (Racine, 1967b, p. 8). Ce numéro étudie différentes manifestations et productions culturelles, dans les domaines de l'art, l'histoire et la politique. Un article en ressort tout particulièrement : " Production culturelle et classes sociales au Québec ", cosigné par Luc Racine, Michel Pichette, Narciso Pizarro et Gilles Bourque, qui le dédicacent ironiquement à Marcel Rioux et Pierre Vadeboncoeur (Racine et al., 1967b). Leur travail se réclame en effet d'une approche scientifique de la culture, qui ne s'embarrasse plus de considérations philosophiques, morales ou esthétiques. Il impressionne par l'ampleur et la rigueur de l'analyse, la justesse du raisonnement, la force des conclusions. Racine souligne d'ailleurs que l'analyse a été élaborée « en tirant parti le plus pleinement possible des travaux d'hommes comme Mehring, Goldman, Lukacs, Marcuse, Althusser et Mache- 
rey » (Racine, 1967b, p. 8). Le marxisme de Parti pris atteint sa maturité. Les jeunes sociologues ont lu Marx ; ils connaissent les différentes interprétations de son œuvre. Ils sont capables de se référer aussi bien à Lukacs et l'école de Francfort qu'à l'école althussérienne ; ils élaborent des problématiques dont les composantes théoriques sont empruntées à l'une comme à l'autre. Mais la revue n'a plus qu'une année à vivre, marquée par de rudes combats politiques qui vont radicaliser sa pensée.

Les numéros de l'automne 1967 et l'hiver 1968 mettent soudainement en scène les opprimés de toutes les régions du monde qui se soulèvent contre l'impérialisme américain. La revue affirme haut et fort que la lutte nationale des Québécois est solidaire de celle des Noirs américains, des Latino-Américains, et des autres peuples engagés dans leur libération. Selon ce point de vue, les États-Unis sont l'ennemi commun, de même que les valets des États-Unis : au Québec, les Anglo-Canadiens et les bourgeois québécois. La lutte nationale est bel et bien une lutte de classes; les doutes que certains ont pu entretenir à cet égard se sont dissipés. "Le nationalisme, écrit Gilles Bourque, s'est changé, au sein des sociétés en voie de décolonisation en une force positive, génératrice de libération pour les opprimés » (Bourque, 1967, p. 10). Au Québec, « l'heure de l'action a définitivement sonné. L'histoire n'attendra pas. L'indépendance, mesure en elle-même indispensable, risque de se faire contre l'intérêt de la majorité des Québécois »(Bourque, 1967, p. 17). La priorité est donc donnée à la lutte politique. Au sein de l'équipe de Parti pris, plusieurs croient en effet que l'indépendance est inévitable mais qu'il faut empêcher la petite bourgeoisie d'en prendre l'initiative. Aussi, faut-il souhaiter « le regroupement des militants socialistes et des intellectuels marxistes dans un mouvement politique » (Racine, 1967c) pour organiser la lutte des travailleurs contre l'impérialisme et ses valets. On ne cesse de répéter que ce « projet révolutionnaire repose sur une analyse véritablement scientifique de la réalité », comme l'écrit, par exemple, Charles Gagnon (Gagnon C., 1968, p. 30). Dans ce contexte, scientifique signifie marxiste. Le ton de la revue devient très combatif et sa ferveur révolutionnaire intense. Ses horizons s'élargissent car l'internationalisme est à l'ordre du jour. Plusieurs auteurs s'intéressent tout particulièrement aux pays de l'Amérique latine. La revue publie un texte de Che Guevara, des articles sur Cuba et sur les républiques andines. Le Che et 
Fidel Castro sont fréquemment cités de même que le président Mao. Le Québec n'est plus seul, parce que « l'envahissement de plus en plus grand de l'impérialisme américain ... vient créer une communauté de destin entre les Québécois et les "damnés de la terre" » (Bourque, 1968, p. 10). L'allusion aux damnés de la terre évoque le titre de l'ouvrage de Frantz Fanon qui a marqué la pensée de Parti pris à ses débuts. Toutefois, la cible de la lutte révolutionnaire West plus le colonialisme mais l'impérialisme.

En 1968, l'information et le débat politique sont prioritaires. Les sociologues deviennent journalistes. Gilles Dostaler réalise deux entrevues remarquables, avec René Lévesque et avec Marcel Pépin. Jan Depocas recueille les propos de François Aquin. La revue présente aussi un reportage sur la situation des Noirs dans la société québécoise. D'autre part, elle organise et anime des tables rondes sur les différentes options politiques de l'heure, au Québec. Une part importante du numéro de l'été 1968 est consacrée au compte rendu d'un grand « débat-forum » sur ces options (Parti pris, 1968). Des professeurs, des écrivains, des artistes, des étudiants y participent ; plusieurs fractions de la gauche sont représentées. Le comité de rédaction est dirigé par Philippe Bernard ; il assume une tâche difficile. En effet, à ce moment, la revue fait face à un dilemme, comme bien d'autres organisations de la gauche. Faut-il appuyer stratégiquement le Mouvement souveraineté-association, fondé par René Lévesque ? Faut-il y participer pour tenter de l'orienter vers la gauche, dans l'espoir que l'indépendance, le cas échéant, débouche sur le socialisme ? Ou bien, au contraire, s'impose-t-il, malgré l'échec du MLP et du PSQ, d'œuvrer sans tarder à la formation d'un parti révolutionnaire ? Ce parti, basé sur la classe ouvrière, réaliserait du même coup l'indépendance et le socialisme, ce dont le MSA est incapable parce qu'il représente la petite bourgeoisie. Au sein de la revue, les avis sont partagés. On apprend que certains membres de la rédaction, partisans de la seconde option, ont démissionné ; les autres membres seraient disposés à continuer. Mais la revue va cesser de paraître. Non sans réaffirmer une dernière fois sa confiance dans le marxisme scientifique, auquel toute la jeune génération marxiste va se rallier, au cours des années à venir. Le marxisme, conçu comme une science, s'oppose à l'idéologie sous toutes ses formes - politique, sociale, morale, religieuse et autres. Il oriente l'action politique et garantit son efficacité. Or, selon Gifles 
Dostaler, l'essai récent de Pierre Vallières, Nègres blancs d'Amérique, va précisément à l'encontre de cette thèse. Dostaler démontre, en effet, que l'auteur est un gauchiste et un idéaliste, son action n'étant pas fondée "sur une analyse scientifique de la réalité québécoise. Il voue, d'ailleurs, une grande admiration au Marx jeune, au Marx idéaliste. Il néglige le Marx mûr, le Marx scientifique [...] De Marx, Vallières retient un schéma simplifié, idéaliste, de l'aliénation, fruit du capitalisme... et une apologie grandiose du grand soir » (Dostaler, 1968, p. 9). Coïncidence révélatrice des divergences au sein de la gauche : un poème de Gaston Miron, accompagné de la dédicace, Pour Pierre Vallières, Charles Gagnon et leurs compagnons, paraît dans ce dernier numéro de Parti pris : " je vous salue clandestins et militants / rebelles avec cause et pour cause / vous tous, connus et inconnus / hommes / plus grands pour toujours que l'âge de vos juges » (Miron, 1968, p. 79). Après sa disparition, la revue Parti pris entre dans l'histoire du Québec, elle devient un mythe. En 1975, Marcel Rioux écrit : «De toutes les publications de gauche de cette période, il semble bien que c'est Parti pris qui a eu la plus grande audience et le plus d'impact sur la société québécoise » (Rioux, 1975, p. 6).

\section{LA REVUE SOCIALISME, 1964-1974}

$\underline{\text { Retour à la table des matières }}$

Quelques mois à peine après la fondation de Parti pris, paraît le premier numéro d'une nouvelle revue, Socialisme 64. Revue du socialisme international et québécois. Fondée par Marcel Rioux et Jacques Dofny, professeurs au Département de sociologie de l'Université de Montréal, la revue sera publiée pendant dix ans ; elle aura une influence considérable. Outre Dofny et Rioux, les professeurs Alfred Dubuc, du Département des sciences économiques, et Michel Van Schendel, du Département des lettres, seront les principaux artisans de Socialisme, jusqu'en 1970. Formé à l'anthropologie culturelle, Marcel Rioux s'est intéressé au marxisme et au socialisme dans les années 1940, lors d'un séjour à Paris. Dans son Essai de sociologie critique, 
en 1978, il affirme privilégier un marxisme culturel qui serait distinct du marxisme économique (Rioux, 1978). Cette version humaniste du marxisme se rapprocherait de la pensée d'Ernst Bloch et de l'école de Francfort. L'influence de Rioux est liée à son enseignement tout autant qu'à ses écrits. De 1961 à 1976, il est titulaire du cours sur Marx et la sociologie marxiste, que tous les étudiants de sociologie doivent suivre obligatoirement. Au début de cette période, les étudiants ne peuvent pas lire Marx et doivent se limiter à des ouvrages comme celui du père Jean-Yves Calvez sur la pensée de Marx (Calvez, 1956). Lucien Goldman et Henri Lefebvre sont invités à parler dans ce cours lorsqu'ils sont de passage à Montréal. Au cours des années 1950, Rioux a participé à la revue Cité Libre ; en 1976, il fonde la revue Possibles qu'il anime avec Gabriel Gagnon. Jacques Dofny est né en Belgique, et il a fait ses études en France. Il a travaillé dans le laboratoire de Georges Friedman, y côtoyant Alain Touraine et Michel Crozier (Bernard, 1995, p. 3). En 1962, il devient professeur au Département de sociologie de l'Université de Montréal. Spécialiste de la sociologie du travail, militant engagé dans l'action syndicale et politique, il n'adhère pas pour autant au marxisme. Sa pensée est proche de celle d'Alain Touraine et des intellectuels socialistes français. Dofny invite à Montréal plusieurs sociologues européens ou américains de gauche ; « il organise un flux incessant de conférenciers étrangers à Montréal », écrit Paul Bernard dans la notice nécrologique de Dofny (Bernard, 1995, p. 3). En outre, 0 favorise la formation en France d'un grand nombre d'étudiants québécois, notamment les jeunes marxistes. Alfred Dubuc a été engagé à l'Université de Montréal, en sciences économiques, au cours des années 1960, à son retour de France où il a fait sa thèse sous la direction de Fernand Braudel. Dubuc applique à l'histoire économique et sociale du Québec et du Canada la grille d'analyse des chercheurs de l'École des Annales, qui s'inspirent en partie du marxisme. Il renouvelle ainsi, à bien des égards, l'interprétation de la rébellion des patriotes et celle du pacte confédératif canadien. À la fin des années 1960, il quitte l'Université de Montréal pour l'UQÀM où il dirigera le Département d'histoire et enseignera jusqu'à sa retraite.

En 1964, le comité de rédaction de la nouvelle revue se compose d'universitaires et de syndicalistes. Ces derniers se rattachent à l'une ou l'autre des grandes centrales syndicales. Le manifeste du premier 
numéro est signé par Émile Boudreau, Jacques Dofny, Roland Martel et Marcel Rioux. Il ne se réclame pas du marxisme mais du socialisme. Selon les auteurs, le socialisme est une théorie et une pratique forgées depuis le XIXe siècle, se définissant comme une tradition et un mouvement social. Le socialisme signifie le contrôle de l'économie, de la politique et de la culture par le peuple, les travailleurs, et aussi le contrôle de leurs vies. Il vise à « faire du Québec une véritable démocratie économique et politique » (Boudreau et al., 1964, p. 11). Il veut " résister au capitalisme exploiteur, aux inégalités sociales, au chômage, à la misère et à l'avilissement de la personne humaine » (Boudreau et al., 1964, p. 12). De plus, le socialisme « fait appel à une solidarité qui transcende les frontières » (Boudreau et al., 1964, p. 22). En 1965, l'éditorial du numéro 6 de la revue, signé par Gérald Fortin, insiste tout particulièrement sur la montée du socialisme et l'existence d'un mouvement mondial de libération à l'égard du capitalisme auquel le Québec n'échappe pas (Fortin, 1965). La revue proclame sa foi en l'unité des trois principes : nationalisme, démocratie, socialisme, qui progressent partout sur la planète, selon l'éditorialiste.

$\mathrm{Au}$ cours des premières années, Socialisme publie peu d'articles théoriques. Ses collaborateurs s'intéressent à la situation sociale et politique dans différents pays. Par exemple, aux États-Unis où la lutte des Noirs retient l'attention, en Israël, dans les Antilles, en particulier à Cuba. La revue ne néglige pas pour autant la conjoncture québécoise. Elle publie des témoignages sur la condition ouvrière, sur des accidents de travail, des conflits et des grèves. D'autre part, elle mène une réflexion approfondie sur les politiques sociales et économiques qu'il conviendrait d'établir au Québec. Les auteurs mettent l'accent, d'une part, sur le développement régional, dans le prolongement des travaux du BAEQ, et, d'autre part, sur les politiques familiales dont Jacques Henripin, professeur de démographie, devient le spécialiste attitré au sein de l'équipe. Le syndicalisme, au Québec et dans d'autres sociétés, est le sujet de plusieurs articles, certains signés par des leaders syndicaux influents : Jean Gérin-Lajoie, Marcel Pépin, Pierre Vadeboncoeur et d'autres. Plusieurs textes s'intéressent à la diffusion du syndicalisme dans de nouveaux secteurs - les emplois de bureau, la fonction publique, les universités -, à sa place dans la société et à son engagement politique. Des sociologues et des politologues de l'Université Laval, en particulier Fernand Dumont, Gérald Fortin, Vincent Le- 
mieux, collaborent régulièrement à Socialisme. Certains participent au comité de rédaction, qui s'élargit au fil des années.

Une part importante du contenu de la revue est consacrée à la recension de revues et de livres publiés en France principalement, mais aussi aux États-Unis. En général, ces publications émanent d'universitaires et de militants qui se situent dans la mouvance de la gauche socialiste. Cette gauche couvre un large éventail de tendances et la revue fait preuve d'éclectisme dans ses lectures. Elle fait écho, par exemple, à des ouvrages de Jacques Berque, Henri Desroches, Daniel Guérin, Georges Friedman, Serge Mallet, Henri Lefebvre. Elle rend compte des premières publications du nouveau mouvement féministe, celles de Betty Friedan et d'Andrée Michel. Le rapport entre le socialisme et le christianisme est un thème privilégié. Par exemple, l'expérience des prêtres-ouvriers en France retient l'attention. L'abbé Jacques Grand'Maison et le père Vincent Harvey signent des textes dans la revue. Certains collaborateurs, en particulier des syndicalistes comme Pierre Vadeboncoeur et Marcel Pépin, s'inspirent d'une philosophie catholique progressiste. L'expression consacrée " une société bâtie pour l'homme » revient souvent sous leur plume. D'autre part, on peut lire aussi dans Socialisme des articles de Dimitri Roussopoulos, anarchiste notoire, qui a fondé la revue anglophone Our Generation en 1961, et des articles de Stanley Ryerson, historien marxiste communiste. On y trouve des encarts publicitaires pour la revue torontoise dirigée par Ryerson, The Marxist Quaterly. Les thèses de Rosa Luxembourg sur la révolution aussi bien que les théories de l'autogestion sont l'objet de discussions. La revue présente à ses lecteurs un document signé par Ernesto Guevara et le testament de Togliatti. L'esprit et le style de Socialisme apparaissent clairement dans les communications présentées par ses membres et ses sympathisants lors du colloque de 1967, "Le socialisme au Québec : une utopie ? », organisé par les étudiants, dans le cadre de la faculté des sciences sociales de l'Université de Montréal. Le texte des interventions est reproduit dans la revue (Socialisme 67, 1967a). Les participants énoncent des idées audacieuses pour l'époque, et ils ont parfois des vues contradictoires. Cependant, ils évitent toute attitude dogmatique ou franchement polémique. Cela va bientôt changer. 
Jusqu'en 1968, l'option en faveur du socialisme a laissé largement ouvert le débat sur la conjoncture politique et les moyens d'action à mettre en oeuvre. Les analyses et les positions politiques des auteurs ne s'accordent pas toujours mais la recherche d'un consensus n'apparaît pas comme prioritaire. Ainsi, le rapport entre l'indépendance et le socialisme est conçu de différentes manières, parfois opposées. La définition des forces révolutionnaires, de leurs alliés et de leurs adversaires, est matière à réflexion et spéculation. Par exemple, en 1966, Dubuc, Rioux et Van Schendel se penchent ensemble sur la victoire électorale récente du parti de l'Union nationale, dans « Perspectives d'avenir pour la gauche », texte qui prend la forme d'un entretien (Rioux et al., 1966). Rioux rejette fermement l'hypothèse d'un retour du conservatisme au Québec. Il affirme que la gauche est partout, dans tous les partis et hors des partis. Il croit notamment que ce sont « les jeunes qui réalisent le mieux la jonction entre la gauche nationale et la gauche sociale » (Rioux et al., 1966, p. 10). Selon Dubuc, l'analyse de Rioux donnerait à penser que l'avenir de la gauche est dans le RIN, mais il n'en demeure pas moins que les forces de gauche, bien qu'elles soient partout en grand nombre, refusent de toute évidence l'action électorale. À son avis, elles n'y sont pas prêtes. « Un parti de gauche, affirme Dubuc, ne peut compter sur l'appui des forces de gauche » (Rioux et al., 1966, p. 17). Van Schendel, pour sa part, insiste sur la nécessité d'approfondir d'abord la pensée théorique, fondement de l'action révolutionnaire. "Les cadres théoriques de l'action font défaut, déplore-t-il, il faut en découvrir de nouveaux » (Rioux et al., 1966, p. 25). Lors du colloque de 1967, Van Schendel a déjà énoncé la thèse qu'il juge fondamentale et qu'il continuera de marteler : "Pas de socialisme sans lutte contre l'impérialisme américain » (Van Schendel, 1967, p. 94). Par contre, l'éditorial du numéro 11, en 1967, affirme que le syndicalisme au Québec est le parti d'opposition en quelque sorte (Socialisme 67, 1967b). Cette analyse conduirait à privilégier le PSQ, qui rassemble notamment la gauche des syndicats. Ces divergences politiques au sein de la revue ne feront que s'amplifier par la suite. En 1968, à l'instar de Parti pris, Socialisme va buter contre la pierre d'achoppement que représente le MSA, fondé par René Lévesque et ses partisans. Dans le numéro 14 de 1968, intitulé "Québec libre », certains auteurs insistent sur l'urgence pour les organisations progressistes d'élaborer un programme socialiste qui forcerait le MSA à dépasser ses ambiguïtés. D'autres dénoncent, dans ce contexte, le con- 
servatisme de certaines gens de gauche qui refuseraient, entre autres, de poser la priorité de la lutte contre la domination économique et politique des États-Unis sur la lutte pour l'indépendance nationale. JeanGuy Loranger, jeune professeur au Département des sciences économiques, présente et analyse des données statistiques illustrant le contrôle américain de l'économie (Loranger, 1968). D'autre part, Pamphile Piché annonce que le syndicalisme est au seuil de l'action politique (Piché, 1968), alors que Pierre Vadeboncoeur dénonce les thèses préétablies et les méthodes de la gauche en ce qui a trait aux syndicats (Vadeboncoeur, 1968). Selon lui, la recette de la gauche serait la suivante : on pose un parti politique, les syndicats l'appuient et on prend le pouvoir. Vadeboncoeur pense, bien au contraire, qu'on doit commencer, non par un parti mais par l'action de masse, à l'écart de tout dogmatisme, et en libérant la critique. « La libération, écrit-il, ne peut être que l'effet de la liberté » (Vadeboncoeur, 1968, p. 24). Mais Socialisme vogue vers d'autres horizons.

La fin de l'année 1968 marque un changement d'orientation important. Sous la direction de Michel Van Schendel, Socialisme devient une publication marxiste, consacrée à l'élaboration d'une " théorie et d'un programme socialiste au Québec ", comme l'indique le titre de l'éditorial du numéro 15 (Van Schendel, 1968, p. 5). Ce texte remet en question la validité théorique de la conception du socialisme présentée dans le manifeste de 1964. Il déplore aussi l'éclectisme de la revue. On y annonce, d'autre part, qu'une « équipe, plus nombreuse et rajeunie [...] repart sur un nouveau pied " (Van Schendel, 1968, p. 5). Cette prise de contrôle de la revue serait justifiée par la conjoncture révolutionnaire du moment, en divers points du globe : Vietnam, Tchécoslovaquie, France... Cette conjoncture, écrit l'éditorialiste, " restitue à la pensée de Marx sa durable et radicale nouveauté »" (Van Schendel, 1968, p. 13). Des collaborateurs nouveaux s'intéressent à divers sujets d'actualité : le MSA, les comités de citoyens dans les quartiers populaires, le mouvement étudiant au Québec et dans d'autres pays. Luc Racine présente une analyse originale de la composition de classes du mouvement nationaliste (Racine, 1968). Selon lui, le MSA serait l'organisation politique, non pas de la grande bourgeoisie mais de la petite bourgeoisie québécoise, notamment de ses couches défavorisées par l'expansion de l'impérialisme américain. Cette petite bourgeoisie chercherait à se rallier les couches moyennes salariées relevant de 
l'État, en vue d'un réaménagement constitutionnel. Au cours des années à venir, les sociologues marxistes reprendront inlassablement l'analyse des classes ou des fractions de classes qui seraient le support du nationalisme québécois. Par ailleurs, dans ce premier numéro marxiste de Socialisme, on découvre une critique féministe de l'encyclique Humanae Vitae sur le contrôle de la fécondité (Lagadec, 1968). L'auteur est professeur au Département de philosophie. Des femmes sont membres de la rédaction, ce qui n'est pas courant à l'époque : Céline Saint-Pierre et Louise Malo de Laberbis, professeures au Département de sociologie, Hélène David, assistante de recherche de Jacques Dofny ; d'autres les suivront.

La nouvelle orientation de la revue se confirme et se précise dans le numéro suivant. Michel Van Schendel occupe le poste de rédacteur en chef, nouvellement créé. Plusieurs membres de l'équipe précédente ont quitté la revue : Dofny, Dubuc, Rioux et les syndicalistes de la première heure. Dans un texte liminaire, Van Schendel fait le point sur la situation, il redéfinit l'objectif et les méthodes de la revue. À cette fin, il s'inspire de la Conférence hémisphérique pour mettre fin à la guerre du Vietnam, qui s'est tenue à Montréal, en novembre 1968. La conférence montre « qu'il est juste de partir des réalités internationales et des problèmes tels qu'ils se constituent à l'échelle internationale, pour analyser les questions de système et de structure qui se posent dans une société particulière, la nôtre en l'occurrence, et pour déterminer les formes de lutte les plus appropriées contre le régime " (Van Schendel, 1969a, p. 7). Dans cette optique, il annonce que la revue présentera nombre d'articles « consacrés à l'examen des problèmes internationaux du socialisme et du capitalisme » et d'autres « qui s'efforceront de traiter sous cette lumière des aspects québécois d'une telle recherche » (Van Schendel, 1969a, p. 10). Toutefois, de l'avis du rédacteur en chef, l'équipe de la revue West pas encore prête à relever ce défi. Une longue période de transition lui sera nécessaire pour acquérir les connaissances théoriques qu'exige ce travail, auquel « l'empirisme ne sied guère » (Van Schendel, 1969a, p. 10). L'équipe devra aussi élucider ce qu'il désigne comme ses contradictions et ses faiblesses méthodologiques. Par ailleurs, l'intervention inopinée d'un groupe québécois indépendantiste dans les débats de la Conférence hémisphérique donne à Van Schendel l'occasion d'exprimer sa profonde aversion pour le nationalisme, en particulier dans les rangs de la gauche. 
Aucun terme West trop méprisant pour qualifier l'attitude de ce groupe : " réflexes de déracinement», " psychologie de l'état de siège », « repliement sur soi », " provincialisme colonial », « manifestation d'impuissance » (Van Schendel, 1969a, note 1, p. 8, 9). Le rédacteur en chef n'est pas au bout de ses peines ! Cannée suivante, il soulignera que cinq numéros de la revue ont été consacrés au " travail de réflexion », lequel « a permis de dégager des perspectives théoriques qui viennent affermir nos positions politiques » (Van Schendel, 1969b, p. 3 ). Il est possible de retracer les principales étapes de cette réflexion. En résumé, elle conduit à dégager la problématique du socialisme de son ancrage national, en donnant la priorité absolue à la lutte contre l'impérialisme. L'équipe de la revue y parviendra, non sans difficultés.

Van Schendel explicite sa position dans deux textes du numéro 17 de Socialisme 69. Le liminaire, "Politique de la revue », est signé par le comité de rédaction mais on y reconnaît le style du rédacteur en chef Le Québec y est défini comme « une nation profondément érodée par le néo-impérialisme nord-américain » (Van Schendel, 1969c, p. 5). Dans ce contexte, Socialisme - « la seule revue progressiste qui reste » - repose sur un accord commun pour explorer les voies d'épanouissement au Québec «d'un projet socialiste rigoureux »(Van Schendel, 1969 c, p. 3, 6). Le texte qui suit le liminaire, «Pour une théorie du socialisme québécois, Il », porte la signature de Van Schendel. L'auteur rappelle l'échec récent du PSQ, du MLP, de la gauche du RIN et de la gauche québécoise du NPD. Dès lors, comment expliquer « l'absurdité du postulat socialiste tel qu'il s'est la plupart du temps manifesté au Québec ? (Van Schendel, 1969d, p. 8). La faute, écrit-il, n'en serait-elle pas au " spontanéisme latent », à « l'anti-intellectualisme », " la méfiance à l'endroit de toute réflexion théorique sur les buts et méthodes de l'action socialiste ", " la conscience terrorisée par son peu de prise sur l'histoire », etc. (Van Schendel, 1969d, p. 9). Dans la foulée, il critique sévèrement la position de Parti pris sur la nation, " son adhésion culturaliste et psychologique à la cause de l'indépendance » (Van Schendel, 1969, p. 25). La notion de nation prolétaire, qu'on trouverait notamment chez Vadeboncoeur, West pas épargnée. Elle fausse le rapport entre nation et classes. Pour finir, il s'attaque à l'hypothèse célèbre, énoncée en 1962 par Rioux et Dofny, sur les rapports réciproques entre le nationalisme, qui serait la conscience ethnique propre à la société québécoise, et la conscience des classes au sein 
de cette société. " L'impérialisme est un système de classes ", affirme Van Schendel ; or, « il est impossible de séparer une bourgeoisie "nationale" de l'impérialisme qui la diminue (la vassalise) mais la subventionne pour prix de cette vassalisation » (Van Schendel, 1969d, p. 21). Cet article soulève des objections de la part de certains membres de la revue, qui s'expriment dans le numéro suivant. Céline Saint-Pierre et Dorval Brunelle, dans " Pour un socialisme scientifique québécois », admettent d'abord que les objectifs définis dans l'article de Van Schendel sont « bien posés et non discutables si on s'en tient au niveau théorique où ils ont été envisagés » (Saint-Pierre et Brunelle, 1969, p. $3)$. Ils déclarent toutefois que la revue doit « dépasser cette façon de poser les problèmes pour en arriver à définir un socialisme scientifique qui tienne compte des particularités... de la société québécoise. [... Ainsi sera-t-il possible d'arriver à définir le Québec comme "maillon faible" du capitalisme nord-américain ou encore du capitalisme canadien » (Saint-Pierre et Brunelle, 1969, p. 3). Ces deux auteurs proposent ensuite un programme de travail pour la revue, comportant trois points principaux : la définition et l'analyse des rapports sociaux de production et de leur articulation découlant de la spécificité de l'économie québécoise ; l'exploration des voies susceptibles de conduire à un socialisme québécois ; la définition d'une pratique politique. Enfin, ils rappellent que la revue « se veut un instrument politique au service de l'ensemble des travailleurs » (Saint-Pierre et Brunelle, 1969, p. 6). Dans un article intitulé «Québécitude et socialisme québécois », Jean-Guy Loranger essaie lui aussi de clarifier ce qu'il considère comme ses positions nationalistes par rapport aux positions socialistes de Van Schendel (Loranger, 1969). Loranger veut « justifier les fondements de la nation québécoise sur les plans culturel, social, économique et politique » de même que "les possibilités de la réalisation socialiste de la nation québécoise » (Loranger, 1969, p. 43). Enfin, Charles Gagnon présente sa problématique de la question nationale, dans "Classes et conscience de classes au Québec », qu'il a écrit en 1966, au cours de son emprisonnement (Gagnon C., 1969). Il critique férocement les artisans de la défunte revue Parti pris, qu'il considère comme un groupe petit-bourgeois qui mettait sur le même pied socialisme et nationalisme. Or, le nationalisme, selon Gagnon, est l'idéologie des bourgeois. Néanmoins, la conscience de classe des travailleurs a une dimension nationale. Ils aspirent à la libération nationale et socialiste car les deux sont indissociables. Tous ces textes sont 
motivés par l'urgence de définir une plateforme marxiste pour la revue et leurs auteurs ne s'embarrassent guère de références ; ils s'en tiennent à quelques textes classiques de Marx, Engels et Lénine. Au cours de l'année 1969, la revue publie toutefois un texte théorique, très didactique, de l'économiste trotskyste Ernest Mandel, sur le sous-développement régional produit par le capitalisme (Mandel, 1969). Elle reproduit aussi des notes posthumes de Léon Trotsky, « Les syndicats à l'époque de la décadence impérialiste », diffusées à Paris sous forme de tract (Trotsky, 1969).

Au cours de cette longue année de transition, le comité d'administration et le comité de rédaction de Socialisme se sont renouvelés, de même que la liste de ses collaborateurs. Peu à peu, les jeunes sociologues ont pris toute la place. D'une part, ceux et celles qui sont chargés d'enseignement et de recherche à l'Université de Montréal : Hélène David, Louise Malo, Céline Saint-Pierre et Luc Racine, de même que Marcel Fournier, auteur d'un texte important : " Histoire et idéologie du groupe canadien-français du Parti communiste » (Fournier, 1969) et Paul Bernard, qui organise et analyse pour la revue une table ronde sur la contestation étudiante et les occupations (Bernard, 1969). En 1970, leur collègue Emilio de Ipola se joindra également à la revue. D'autre part, pour former le noyau dur de la nouvelle équipe marxiste, Van Schendel a recruté aussi les jeunes sociologues qui ont participé à Parti pris et leurs camarades. Ils sont professeurs à l'UQ ÀM. Ce groupe se compose de Gilles Bourque, Gilles Dostaler, Nicole LaurinFrenette, Michel Pichette, Jean-Marc Piotte, Narciso Pizarro, auxquels se joindront un peu plus tard Roch Denis, Louis Gill, Suzanna de Ipola et Jorge Niosi. Ils ont obtenu ou ils obtiendront prochainement un doctorat de 3e cycle d'une faculté ou d'une école françaises. Leurs directeurs de thèse sont Charles Bettelheim, Maurice Godelier, Lucien Goldman, Henri Lefebvre, Nicos Poulantzas et d'autres professeurs marxistes. Paul Chamberland, devenu lui aussi professeur à l'UQÀM, publie des poèmes dans la revue ; on peut y lire également Michèle Lalonde, Gaston Miron, Luc Racine et d'autres poètes. À partir de 1970, quelques étudiants en lettres et en sociologie de l'UQÀM participent à la revue : Marcel Chouinard, Madeleine Monette, Paul Rompré. Il n'y a plus de syndicalistes dans le comité de rédaction; les sociologues de la première génération n'en font plus partie. En 1969, Socialisme publie néanmoins deux textes de Marcel Rioux sur l'éduca- 
tion artistique dans la " société post-industrielle et technétronique » (Rioux, 1969a, 1969b). L'auteur s'inspire du résultat des recherches réalisées pour la Commission d'enquête sur l'enseignement des arts au Québec, qu'il a présidée.

Les textes du dernier numéro de l'année 1969 sont représentatifs de la nouvelle production de Socialisme. Ce sont des analyses rigoureuses, fondées sur une problématique théorique très structurée, qui allient la perspective de l'histoire, de l'économie et de la sociologie. Elles font une large place à la critique de travaux théoriques ou empiriques, qui se rattachent au marxisme ou à d'autres courants. Dans « Le syndicalisme contre la révolution ? ", Michel Pichette établit, à partir de plusieurs citations de Marx, que « le syndicat se trouve lui-même inséré dans la structure des rapports de production capitalistes » (Pichette, 1969, p. 14). D'où il conclut que le syndicalisme « allie les travailleurs au système établi » et "retarde... l'émergence de la conscience révolutionnaire »(Pichette, 1969, p. 18). Gilles Dostaler et Luc Racine signent «Contre Marcuse. Essai sur la pensée idéologique dans les sociétés industrielles », une brillante critique du caractère idéaliste et inopérant, selon les auteurs, de l'analyse marcusienne de la société capitaliste (Dostaler et Racine, 1969). « Marcuse ne retient de Marx, écrivent-ils, que les aspects secondaires et dépassés, par Marx lui-même, de sa pensée » (Dostaler et Racine, 1969, p. 45), c'est-à-dire les aspects hégéliens, idéalistes, bref non scientifiques. Les concepts et les hypothèses de Marcuse sont donc récusés en tant qu'ils s'opposent à une approche scientifique. On retrouve dans ce texte la conception du marxisme comme science, mise de l'avant par les jeunes sociologues, dans les derniers numéros de Parti pris. Au demeurant, cette conception aura marqué plusieurs périodes de l'histoire du marxisme, au Québec comme ailleurs. Dans la même veine, un article de Narciso Pizarro, intitulé " Les groupes marginaux ? Idéologie et réalité », démontre au terme d'une critique approfondie le caractère idéaliste de la sociologie de l'action de Talcott Parsons, un idéalisme que partageraient les théories de la société industrielle de Touraine, Aron, Galbraith et d'autres penseurs (Pizarro, 1969). L'auteur soutient que les concepts clé de ces théories - l'action, l'acteur et le système social permettent de définir la marginalité dans les termes de ce qu,il appelle l'idéologie américaine, celle de la classe dominante. 
En 1970, la revue s'engage dans la dernière étape de son parcours. Elle adopte le nouveau nom de Socialisme québécois, ce qui voudrait « indiquer une rupture critique » (Van Schendel, 1970, p. 2), selon l'éditorial du numéro 20, qui est à l'évidence du rédacteur en chef, même s'il ne l'a pas signé. Il s'agit, écrit-il, de rompre avec un « éclectisme », de quitter le " no mans land d'un "dialogue" où tout le monde aurait droit de parole, pourvu qu'il soit animé de... bonnes intentions » (Van Schendel, 1970, p. 2). Désormais, « l'exercice d'une rigueur sans concessions deviendra la seule garantie et la seule justification de la pertinence et de l'efficacité de notre entreprise » (Van Schendel, 1970, p. 2). Faisant appel « aux principes du marxisme-léninisme, seuls capables d'informer une théorie et une pratique politique révolutionnaire », l'éditorialiste s'efforce de montrer la réciprocité entre la pratique théorique et la pratique politique, dans l'optique althussérienne (Van Schendel, 1970, p. 2). Pour terminer, il propose une interprétation des différentes conjonctures québécoises depuis le XIXe siècle, en fonction de l'impérialisme sous sa forme ancienne et nouvelle, et de son effet sur le Québec et le Canada. Plusieurs membres de l'équipe vont alors privilégier l'analyse de conjonctures historiques. Il s'agit, en quelque sorte, de relire l'histoire sous un nouveau jour. Dans ce numéro 20, la revue publie un texte de Gilles Bourque et Nicole LaurinFrenette, "Classes sociales et idéologies nationalistes au Québec, 1760-1970 » (Bourque et Laurin-Frenette, 1970). Ils soutiennent la thèse selon laquelle, à chacune des étapes charnières de l'histoire du Québec, les classes et les fractions de classe ont élaboré une forme particulière du discours nationaliste, celle qui correspondait à leur place dans l'économie et à leur intérêt politique. Cette étude s'appuie sur une théorie de la nation, inspirée de la conception althussérienne du politique, et notamment des travaux de Nicos Poulantzas. Les auteurs engagent une vive polémique à propos des thèses de Fernand Dumont, Jacques Dofny et Marcel Rioux sur la nation et l'idéologie nationaliste. Ces thèses, jugées idéalistes, sont sévèrement critiquées. £analyse insiste par ailleurs sur la place et le rôle des petites bourgeoisies dans diverses conjonctures. Les auteurs en identifient plusieurs : traditionnelle, clérico-bourgeoise, libérale, néocapitaliste, technocratique... Elles continueront de proliférer dans les travaux marxistes sur la société québécoise, en changeant sans cesse de nom et parfois d'allégeance. L'article de Bourque et Frenette sera repris l'année suivante dans la revue, sous le titre « La structure nationale québécoi- 
se »(Bourque et Laurin-Frenette, 1971). Il aura été modifié de manière à prendre en compte les événements survenus au Québec lors de la crise d'octobre 1970. Dans ce contexte, les auteurs écrivent que l'idéologie révolutionnaire des classes dominées au Québec pourrait « être à la fois anti-bourgeoise, anti-impérialiste et nationaliste » (Bourque et Laurin-Frenette, 1971, p. 153). Leur libération resterait néanmoins entièrement liée à celle du prolétariat nord-américain dans son ensemble. Les analyses de conjoncture s'appliquent à faire ressortir les effets de l'impérialisme sur le Québec et le Canada. Elles insistent aussi sur l'urgence et la nécessité d'une organisation politique propre à la classe ouvrière. Ainsi, l'éditorial du numéro 21-22, consacré à la crise d'octobre 1970, retrace l'évolution du Québec depuis les années 1960, afin de démontrer que les rapports internes de domination et celle qu'exerce la société anglo-canadienne sur le Québec, " sont directement fonction de l'évolution propre de la structure de domination qu'exerce l'impérialisme américain sur l'ensemble du Canada (Québec compris) » (Socialisme québécois, 1971, p. 5). Dans ce contexte, le FLQ da pas été la cause de la répression pas plus que la gauche québécoise n'en a été la cible. La bourgeoisie canadienne et la bourgeoisie québécoise ont déployé leur arsenal répressif contre la classe ouvrière pour " se maintenir comme classe-relai vis-à-vis du capital américain »(Socialisme québécois, 1971, p. 13). L'éditorial conclut à la nécessité, en contexte d'affrontement entre les classes, d'une « organisation dirigée et contrôlée par les travailleurs »(Socialisme québécois, 1971, p. 16). Celle-ci aurait la tâche de coordonner, fusionner, centraliser les luttes et les diriger contre l'État bourgeois. Dans la même livraison de la revue, Luc Racine et Roch Denis publient « La conjoncture politique québécoise depuis $1960 »$, un travail remarquable, d'une large envergure (Denis et Racine, 1971). Les auteurs récapitulent d'abord l'évolution des classes et de leurs fractions sur le plan politique, en fonction des événements et des conditions économiques. Cette partie de l'étude démontre notamment que l'impérialisme américain est toujours le facteur déterminant. Elle est suivie d'une histoire des idées, des groupes nationalistes, des groupes socialistes et du mouvement étudiant. Les auteurs expliquent les positions de ces différentes formations et font la critique de leurs luttes. Dans leur post mortem de Parti pris, ils reprochent à la revue ses errements sur la question nationale. En conclusion, Denis et Racine constatent que le mouvement socialiste est constitué de noyaux dispersés, qui devraient être intégrés 
au mouvement ouvrier, " au fur et à mesure que les travailleurs marchent vers la construction de leur organisation politique indépendante » (Denis et Racine, 197 1, p. 78). Ce numéro de 1971 se termine par un texte de Michel Van Schendel, "Impérialisme et classe ouvrière au Québec » (Van Schendel, 1971). L'auteur étudie les formes de la domination impérialiste sur la société québécoise dont dépend la définition même de la classe ouvrière. Il s'appuie, d'une part, sur Lénine et Rosa Luxembourg qu'il cite abondamment, et, d'autre part, sur les économistes marxistes qui font autorité : Baran et Sweezy, Bettelheim, Gunder Franck, Pailloix, Samir Amin.

Le numéro suivant, «Un maillon de la chaîne 》, est presque entièrement consacré à des questions de théorie et d'analyse économiques. Ces questions apparaissent comme prioritaires dès lors que l'accent est placé sur la lutte contre l'impérialisme. Gilles Bourque, le nouveau rédacteur en chef de la revue, le confirme d'ailleurs dans l'avant-propos, un texte non signé qu'il a publié plus tard dans une anthologie. Il écrit que « durant les années 1960, l'une des principales faiblesses des textes et des revues se réclamant du marxisme était sans doute l'absence de tentative sérieuse d'analyse de l'instance économique. [...] Presque tous nageaient dans le nationalisme de gauche... Or, dans cette problématique, il n'est pas innocent que l'étude de l'infrastructure soit absente - elle doit l'être » (Bourque, 1972a, p. 7). Et il conclut par cette phrase lapidaire, qui deviendra célèbre : «Malheureusement le marxisme ne se traite pas à la sauce nationaliste, fusse-t-on le petit-bourgeois le plus radical qui soit. Toutes les proclamations de foi marxiste qu'on voudra faire n'y suffiront pas » (Bourque, 1972, p. 8). Dans ce dossier sur l'économie, Louis Gill explique le lien entre la croissance et l'asservissement (Gill, 1972), et Dorval Brunelle propose des éléments pour une théorie de l'impérialisme (Brunelle, 1972). On y trouve aussi un texte pédagogique de Claude Saint-Onge sur la baisse tendancielle du taux de profit (Saint-Onge, 1972). Ces auteurs s'attaquent à des problèmes difficiles; ils tentent d'expliciter des notions marxistes complexes, parfois obscures, et de les appliquer à la situation économique internationale ou locale. En fin de numéro, on peut lire un texte politique de Gilles Bourque, " En réponse à Pierre Vallières », une critique virulente de la prise de position de l'ex-felquiste en faveur du Parti Québécois (Bourque, 1972b). Cette position, selon Bourque, se situe dans la logique de l'action menée par le FLQ, « qui a 
toujours été pratiquée comme celle d'une aile clandestine des organisations nationalistes légales » (Bourque, 1972b, p. 135). Il récuse les notions de «nation prolétaire » et de «bourgeoisie americano-canadian » employées par Vallières et condamne les stratégies qu'il prône à l'endroit du Parti Québécois : l'entrisme et l'étapisme. " Le militant de "gauche" qui entre au PQ, écrit Gilles Bourque, retarde l'organisation de la classe ouvrière et permet une imprégnation plus grande de l'idéologie d'une classe adverse sur le prolétariat " (Bourque, 1972b, p. 137). Vallières n'aura donc jamais trouvé grâce devant la gauche marxiste.

Le dernier numéro de la revue paraît en 1974. Un intervalle de deux ans le sépare du numéro précédent. Entre-temps, le comité de rédaction s'est dispersé ; il. ne reste que Gilles Bourque et Céline Saint-Pierre ; celle-ci est devenue professeure à l'UQÀM. Jean-Marc Piotte reprend le débat sur la question nationale là où Gifles Bourque l'a laissé, en 1972, dans sa réponse à Vallières. Piotte radicalise le raisonnement de Bourque. En effet, il affirme que le Parti Québécois n'est pas la solution car l'indépendance ne peut être une étape vers le socialisme et « la lutte de la bourgeoisie québécoise contre la bourgeoisie canadienne ne peut que renforcer la domination de l'impérialisme américain sur le Canada et sur le Québec »(Piotte, 1974, p. 6). «Il faut bien se le mettre dans la tête, écrit-il, il n'y aura pas de socialisme québécois »(Piotte, 1974, p. 7), et plus explicitement encore : « aucune libération, aucune révolution n'est possible ici sans que l'impérialisme américain soit détruit chez lui, en son sein » (Piotte, 1974, p. 7). Par conséquent, la priorité doit être donnée à la lutte contre l'impérialisme, partout dans le monde. Les idées politiques des jeunes sociologues ont beaucoup évolué depuis l'époque de Parti pris. D'autre part, ils forment désormais une école de pensée dans le champ de la sociologie. Ce dernier numéro de Socialisme québécois le montre clairement. Axé principalement sur des questions de théorie, il représente à cet égard une contribution importante à la sociologie marxiste. Un texte de Céline Saint-Pierre, « De l'analyse marxiste des classes dans le mode de production capitaliste ", s'attaque au problème de la définition des classes, en partant de l'oeuvre de Marx, celle de Lénine, des travaux récents de Nicos Poulantzas et des intellectuels du Parti communiste français (Saint-Pierre, 1974). En 1971, ces derniers ont publié un ouvrage collectif d'économie politique, Le capitalisme monopoliste 
d'État, qui obtient une large audience dans la gauche québécoise. Selon Saint-Pierre, la définition du travail improductif est particulièrement cruciale parce qu'elle permet de déterminer la place et le rôle de la petite bourgeoisie dans le mode actuel de production capitaliste. L'auteure propose une définition inédite de cette classe, qui met l'accent sur les fonctions de direction politique et idéologique qu'elle assume dans la gestion et la reproduction des rapports sociaux. Elle identifie en outre différentes fractions de la petite bourgeoisie. Sur le plan politique, cette classe oscillerait entre la classe dominante et la classe ouvrière mais pencherait plus souvent vers la première. Trois tableaux complètent le texte ; ils permettent de récapituler et de comparer la théorie des classes de Poulantzas, celle du PCF et celle de Saint-Pierre. Jorge Niosi s'intéresse lui aussi à l'analyse marxiste des classes, dans un texte intitulé «Pour l'analyse socio-économique de la classe propriétaire » (Niosi, 1974). Il dresse d'abord une typologie des fractions économiques de cette classe. Partant des travaux d'Ossowski et de Poulantzas, il dégage ensuite les variables qu'il considère comme les plus importantes pour établir des distinctions entre les fractions : la nationalité du capital, sa taille, le secteur d'investissement, et autres. L'auteur démontre aussi que cette typologie est nécessaire à la compréhension du jeu de la classe propriétaire et de ses fractions sur la scène politique. Un article de Nicole Laurin-Frenette, « La libération des femmes », examine le cadre théorique dont s'inspirent les principaux courants du nouveau mouvement féministe : le marxisme (Engels, Lénine, Bebel), la psychanalyse (Reich), l'existentialisme (Beauvoir) et les diverses versions du féminisme révolutionnaire en France, en Angleterre et aux États-Unis (Laurin-Frenette, 1974). L'auteure se propose de faire une synthèse de ces différents points de vue en les resituant dans une théorie de la reproduction. Elle s'efforce aussi de dégager leurs implications pratiques. Madeleine Gagnon, écrivaine et professeure de littérature à l'UQÄM, signe « Productions culturelles et luttes des classes » (Gagnon M., 1974). En s'appuyant sur les thèses de Marx et Althusser, elle explicite les conditions requises pour une théorie et une analyse de la production culturelle qui ne se dissocie pas d'une pratique politique de transformation. Ce numéro de Socialisme québécois aura une diffusion qui da pas de précédent dans l'histoire de la revue. Certains articles seront reproduits dans des anthologies, et fréquemment cités pendant plusieurs années, au Québec et ailleurs. Ils seront utilisés dans l'enseignement universitaire et dans la 
formation militante, dispensée par les syndicats et les groupes de gauche.

Au cours des années suivantes, plusieurs tentatives pour relancer la revue Socialisme vont échouer. En 1975, un groupe d'intellectuels et d'artistes fondent la revue Chroniques, qui sera publiée jusqu'en 1978. Plusieurs sont marxistes, notamment Céline Saint-Pierre, Madeleine Gagnon et Jean-Marc Piotte. En 1978, la revue Les cahiers du socialisme paraît à l'UQÀM ; Gilles Bourque est le premier directeur. On y retrouve plusieurs anciens collaborateurs de Parti pris et de Socialisme : Dorval Brunelle, Roch Denis, Gifles Dostaler, Alfred Dubuc, Louis Gill, Jorge Niosi, Jean-Marc Piotte et autres. Après la fin des Cahiers, en 1984, il n'y a plus de revue marxiste. Au Québec comme ailleurs, la conjoncture politique West pas favorable aux idées révolutionnaires, et les sciences sociales se désintéressent peu à peu du marxisme.

\section{RÉSUMÉ}

\section{Retour à la table des matières}

Deux départements de sociologie assurent au premier chef le développement et l'essor du marxisme dans le Québec francophone : le département de l'Université de Montréal, au cours des années 1960, relayé par celui de l'Université du Québec à Montréal, au cours des années 1970, Pour retracer la genèse de cette sociologie marxiste, deux revues serviront de fil conducteur : Parti pris et Socialisme. On retracera d'abord le parcours de Parti pris, de 1963 à 1968. Elle peut être considérée comme le berceau de la jeune génération de marxistes. On suivra ensuite l'évolution de la revue Socialisme, au cours de ses dix années de publication. Fondée en 1964 par la première génération de sociologues marxistes ou marxisants, elle tombe quatre ans plus tard entre les mains de la jeune génération, qui la dirige jusqu'à 1974. Elle contribuera à la formation d'une école de pensée originale, axée sur la théorie et l'analyse de la question nationale et des classes sociales. 


\begin{abstract}
Two sociology departments predominated in assuming the development and spread of Marxism in francophone Quebec : the sociology department of the Université de Montréal during the 1960s, followed by that of the Université du Québec àMontréal, during the 1970s. To retrace the origins of this Marxist sociology, two journals will serve as a lead : Parti pris and Socialisme. We shall first of all examine the development of Parti pris, from 1963 to 1968. It may be considered as the cradle of the young generation of Marxists. We shall then study the evolution of the journal Socialisme, during its ten years of publication. Founded in 1964 by the first generation of Marxist or Marxist-oriented sociologists, it passed four years later into the hands of the young generation who edited it until 1974. It would contribute to the creation of a school of original thought, based on the theory and analysis of the national question and social class.
\end{abstract}

\title{
RESUMEN
}

Dos departamentos de sociología garantizan en primer lugar el desarrollo y el auge del marxismo en el Quebec firancófono : el departamento de la Universidad de Montreal, durante los años sesenta, sustituido por el de la Universidad de Quebec en Montreal, durante los años setenta. Para describir la génesis de esta sociología marxista, dos revistas servirán de hilo conductor : Partis Pris y Socialisme. Se describirá en primer lugar el curso de Parti Pris, de 1963 a 1968. Puede considerarse como la cuna de la joven generación de marxistas. Se seguirá a continuación la evolución de la revista Socialisme, durante sus diez años de publicación. Fundada en 1964 por la primera generación de sociólogos marxistas o marxisantes, cae cuatro años más tarde entre las manos de la joven generación, que la dirige hasta 1974. Contribuirá a la formación de una escuela de pensamiento original, orientada hacia la teoría y el análisis de la cuestión nacional y de las clases sociales. 
$\underline{\text { Retour à la table des matières }}$

BEAUDIN, R., " Critique des fondements théoriques de la stratégie anarchiste », Parti pris, vol. 3, no 6, janvier 1966, pp. 9-23.

BERNARD, P., « Qu'est-ce que la contestation a contesté ?, Socialisme 69, no 16, janvier-février-mars 1969, pp. 85-108.

BERNARD, Paul, « Jacques Dofny, un homme d'espoir », Sociologie et sociétés, vol. 27, no 1, printemps 1995, pp. 3-4.

BOUDREAU, É., Jacques DOFNY, ROLAND MARTEL et Marcel Rioux, "Matériaux pour la théorie et la pratique d'un socialisme québécois », Socialisme 64, no 1, printemps, pp. 5-23.

BOURQUE, Gilles, " De Gaulle, politique et stratégie », Parti pris, vol. 5, no 1, septembre 1967, pp. 7-17. BOURQUE, Gilles, « On n'est pas le Congo », Parti pris, vol. 5, no 5, février 1968, pp. 10- 11. BOURQUE, Gilles, "Avant-propos », Socialisme québécois, no 23, 1972-1973, pp. 5-9. BOURQUE, Gilles, « En réponse à Pierre Vallières », Socialisme québécois, no 23, 1972-1973, pp. 127-138. BOURQUE, Gilles et Luc RACINE, « Histoire et idéologie », Parti pris, vol. 4, no 5-6, janvier-février 1967, pp. 33-51.

BOURQUE, Gilles et Nicole LAURIN-FRENETTE, « Classes sociales et idéologies nationalistes au Québec, 1760-1970 », Socialisme québécois, no 20, avril-mai-juin 1970, pp. 13-55. [Texte disponible dans Les Classiques des sciences sociales. JMT.]

BOURQUE, Gilles et Nicole LAURIN-FRENETTE, « La structure nationale québécoise ", Socialisme québécois, no 21-22, 1971, pp. 109-155. [Texte en préparation pour Les Classiques des sciences sociales. JMT.] 
BRUNELLE, Dorval, «L'impérialisme : éléments pour une théorie », Socialisme québécois, no 23, 1972-1973, pp. 57-78.

BRUNELLE, Dorval et Céline SAINT-PIERRE, « Pour un socialisme scientifique québécois », Socialisme 69 , no 18, juillet-août-septembre 1969, pp. 42-58. [Texte en préparation pour Les Classiques des sciences sociales. JMT.]

CALVEZ, J.-Y. (1956), La pensée de Karl Marx, Paris, Éditions du Seuil, 1956.

CHAMBerland, P., " La révolution, c'est le peuple », Parti pris, vol. 2, no 8, mai 1964, pp. 2-10.

CHAMBERLAND, P., « Éditorial : Exigences théoriques d'un combat politique ", Parti pris, vol. 4, no 1, septembre-octobre 1966, pp. 2-10.

CHAMBERLAND, P. et al. (dir.) " Notes biographiques », in Poésie et politique. Mélanges offerts en hommage à Michel Van Schendel, Montréal, L'Hexagone, pp. 481-483.

DENIS, Roch et Luc RACINE, « La conjoncture politique québécoise depuis 1960 », Socialisme québécois, no 21-22, 1971, pp. 17-78.

DOSTALER, Gilles, « Nègres blancs d'Amérique », Parti pris, vol. 5. no 8-9, été 1968, pp. 8-9.

DOSTALER, Gilles, «Économie, littérature et politique. Parcours croisés », in Chamberland, Paul et al. (dir.), Mélanges offerts à Michel Van Schendel, Montréal, L'Hexagone, 2001, pp. 463-472.

DOSTALER, Gilles et Luc RACINE (1969), « Contre Marcuse », Socialisme 69, no 19, octobre-novembre-décembre 1969, pp. 39-62.

FORTIN, Gérald, «Éditorial : La montée du socialisme », Socialisme 65, no 6, printemps 1965, pp. 5-10. [Texte disponible dans Les Classiques des sciences sociales. JMT.] 
FOURNIER, Marcel, « Histoire et idéologie du groupe canadienfrançais du Parti communiste, 1925-1945 », Socialisme 69, no 16, janvier-février-mars 1969, pp. 63-78.

GAGNON, Charles, « Pourquoi la révolution? », Parti pris, vol. 5, no 5, février 1968, pp. 27-33.

GAGNON, Charles, "Classes et conscience de classe ", Socialisme 69, no 18, juillet-août-septembre 1969, pp. 66-74.

GAGNON, G., " Pour un socialisme décolonisateur », Parti pris, vol. 4, no 1, septembre-octobre 1966, pp. 40-55.

GAGNON, G., « Les voies de l'autogestion », Parti pris, vol. 4, no 7-8, mars-avril 1967, pp. 56-72.

GAGNON, M., "Productions culturelles et luttes des classes », Socialisme québécois, no 24, 1974, pp. 63-77.

GILL, L., «Croissance économique et asservissement », Socialisme québécois, no 23, 1972, p. 11-32.

KOSTAKEFF, G., " Marxisme et créditisme », Parti pris, vol. no 8, mars 1966, pp. 33-51.

LAGADEC, Claude, "Le grand-prêtre et la pilule », Socialisme 68, no 15, octobre-novembre-décembre 1968, pp. 116-123.

LAURIN-FRENETTE, Nicole, "La libération des femmes », Socialisme québécois, no 24, 1974, pp. 47-62. [Texte disponible dans Les Classiques des sciences sociales. JMT.]

LIMOGES, C., «Éditorial : De l'homo politicus à nous », Parti pris, vol. 1, no 9-10-11, été 1964, pp. 2-5.

LORANGER, J.-G., " L'impérialisme américain au Québec, analyse de données récentes », Socialisme 68, no 14, 1968, pp. 26-51. 
LORANGER, J.-G., «Québécitude et socialisme québécois », Socialisme 69, no 18, juillet-août-septembre 1969, pp. 42-58.

MAHEU, P., « La protection de l'État », Parti pris, vol. 3, no 1-2, octobre-novembre 1965, pp. 6-15.

MANDEL, E., « Capitalisme et économie régionale », Socialisme 69, no 17, avril-mai-juin 1969, pp. 28-40.

MILL, M., « Sur la théorie de Gorz », Parti pris, vol. 3. no 1-2, octobre-novembre 1965, pp. 6-15.

MIRON, Gaston, « Le salut d'entre les jours » (extrait), Parti pris, vol. 5, no 8-9, été 1968, pp. 79.

NIOSI, Jorge, « Pour l'analyse socio-économique de la classe propriétaire », Socialisme québécois, no 24, 1974, pp. 41-46.

PARTI PRIS, « Éditorial : Parti pris, le RIN et la révolution », vol. 1, no 3, décembre 1963, pp. 2-6.

PARTI PRIS, « Éditorial : Nous avons choisi la révolution », vol. 1, no 5, février 1964, pp. 2-5.

PARTI PRIS , « Bilan du cléricalisme », vol. 4, no 3-4, novembredécembre 1966.

PARTI PRIS, « Les syndicats entre le néo-capitalisme et l'autogestion », vol. 4, no 7-8, mars-avril 1967.

PARTI PRIS, «Aliénation et dépossession », vol. 4, no 9-10-11-12, mai-août 1967.

PARTI PRIS, "Québec'68 / Débat forum », vol. 5, no 8-9, été 1968.

PICHÉ, P. (1968), « Le syndicalisme au seuil de l'action politique $»$, Socialisme 68, no 14, pp. 8-18. 
PICHETTE, Michel, « Le syndicalisme contre la révolution ? », Socialisme 69, no 19, octobre-novembre-décembre, pp. 11-18.

PIOTTE, Jean-Marc, «Éditorial : Le socialisme », Parti pris, vol. 1, no 6, mars 1964, pp. 2-4.

PIOTTE, Jean-Marc, « Autocritique de Parti pris », Parti pris, vol. 2. no 1, septembre 1964, pp. 36-44.

PIOTTE, Jean-Marc, « Où allons-nous ? », Parti pris, vol. 3, no 12, août-septembre 1965, pp. 64-85.

PIOTTE, Jean-Marc, « Le traité d'économie marxiste de Mandel », Parti pris, vol. 3, no 1-2, août-septembre 1965, pp. 108-109.

PIOTTE, Jean-Marc, « Éditorial : Individu, situation et morale », Parti pris, vol. 3, no 8, mars 1966, pp. 2-5.

PIOTTE, Jean-Marc, "Une question de stratégie », Socialisme québécois, no 24, 1974, pp. 5-9.

PIZARRO, N., « Les groupes marginaux : idéologie et réalité. 2e partie : L'idéologie américaine, Socialisme 69 , no 19, octobre-novembre-décembre 1969, pp. 63-75.

POLLENDER, A., " La réforme de la planification en URSS », Parti pris, vol. 3, no 5, décembre 1965, pp. 43-54.

RACINE, Luc, «Politique et théorie », Parti pris, vol. 4, no 5-6, janvier-février 1967, pp. 89-94.

RACINE, Luc, " Présentation : Dépossession et domination ", Parti pris, vol. 4. no 9-10-11-12, mai-août 1967, pp. 7-11.

RACINE, Luc, « Les essais », Parti Pris, vol. 5, no 1, septembre 1967, pp. 49-54. 
RACINE, Luc, « Les mouvements nationalistes au Québec et la lutte pour le socialisme ", Socialisme 68, no 15, octobre-novembredécembre 1968, pp. 37-46.

RACINE, Luc, Narciso PIZARRO, Michel PICHETTE et Gilles BOURQUE, " Production culturelle et classes sociales au Québec ", Parti pris, vol. 4, no 9-10-11-12, mai-août 1967, pp. 43-75.

RIOUX, Marcel, « Du Rapport Parent à la société technétronique », Socialisme 1969, no 17, avril-mai-juin 1969, pp. 60-66.

RIOUX, Marcel, « L'éducation artistique et la société post-industrielle », Socialisme 69, no 19, octobre-novembre-décembre 1969, pp. 93-101.

RIOUX, Marcel, « Remarques sur le phénomène Parti pris », in Joseph Bonenfant (dir.), Index de Parti pris 1963-1968, Sherbrooke, Centre d'étude des littératures d'expression française, 1975, pp. 3-9.

RIOUX, Marcel, Essai de sociologie critique, Montréal, Hurtubise HMH, 1978.

RIOUX, Marcel, Alfred DUBUC et Michel VanSCHENDEL, « Entretien : perspectives d'avenir pour la gauche », Socialisme 66, no 9, octobre-novembre-décembre 1966, pp. 7-25.

RYERSON, STANLEY B., «La pensée de Marx au Canada », Cité Libre, vol. XV, no 46, 1965, pp. 17-22.

SAINT-ONGE, C., « La baisse tendancielle du taux de profit (texte pédagogique) », Socialisme québécois, no 23, 1972-1973, pp. 33-56.

SAINT-PIERRE, C., « De l'analyse marxiste des classes dans le mode de production capitaliste ", Socialisme québécois, no 24, 1974, pp. 9-33. [Texte disponible dans Les Classiques des sciences sociales. JMT.]

SOCIALISME 67, « Le socialisme au Québec : une utopie ? », no 12-13, avril-mai-juin 1967. 
SOCIALISME 67, « Éditorial : Le deuxième souffle de la révolution québécoise », no 11, février-mars 1967, pp. 3-13.

SOCIALISME QUÉBÉCOIS, « Éditorial : La crise d'octobre au Québec », no 21-22, 1971, pp. 5-16.

TROTSKY, Léon, « Les syndicats à l'époque de la décadence impérialiste », Socialisme 69, no 19, octobre-novembre-décembre 1969, pp. 25-32.

TRUDEL, J. (1965), « Éditorial : La crise de l'AGEUM, révolution ou gauchisme? », Parti pris, vol. 3, no 5, décembre, pp. 2-5.

VADEBONCOEUR, Pierre, " Méditation aventureuse sur les méthodes de la gauche », Socialisme 68, no 14, 1968, pp. 19-25.

VAN SCHENDEL, Michel, " La maladie infantile du Québec », Parti pris, vol. 1, no 6. mars 1964, pp. 25-45.

VAN SCHENDEL, Michel, "Pas de socialisme sans lutte contre l'impérialisme américain ». Socialisme 67, no 12-13, avril-mai-juin 1967, pp. 94-111.

VAN SCHENDEL, Michel, «Éditorial : Pour une théorie et un programme socialiste au Québec », Socialisme 68, no 15, octobre-novembre-décembre 1968, pp. 3-18.

VAN SCHENDEL, Michel, « La Conférence hémisphérique est un modèle à suivre ", Socialisme 69, no 16, janvier-février-mars 1969, pp. 7-10.

VAN SCHENDEL, Michel, " Note de la rédaction », Socialisme 69, no 19, octobre-novembre-décembre 1969, pp. 3.

VAN SCHENDEL, Michel, "Politique de la revue », Socialisme 69, no 17, avril-mai-juin 1969, pp. 3-6. 
VAN SCHENDEL, Michel, « Pour une théorie du socialisme québécois Il », Socialisme 69, no 17, avril-mai-juin 1969, pp. 7-26.

VAN SCHENDEL, Michel, «Éditorial», Socialisme québécois, no 20, avril-mai-juin 1970, pp. 2-12.

VAN SCHENDEL, Michel, «Impérialisme et classe ouvrière au Québec », Socialisme québécois, 1970, no 21-22, pp. 156-209.

Fin du texte 